\title{
Pattern Formation in a Diffusive Ratio-Dependent Holling-Tanner Predator-Prey Model with Smith Growth
}

\author{
Bo Yang \\ School of Electronic and Information Engineering, Gansu Lianhe University, Lanzhou 730000, China \\ Correspondence should be addressed to Bo Yang; yangbo_20130202@163.com \\ Received 3 February 2013; Accepted 4 March 2013 \\ Academic Editor: Yonghui Xia \\ Copyright (C) 2013 Bo Yang. This is an open access article distributed under the Creative Commons Attribution License, which \\ permits unrestricted use, distribution, and reproduction in any medium, provided the original work is properly cited.

\begin{abstract}
The spatiotemporal dynamics of a diffusive ratio-dependent Holling-Tanner predator-prey model with Smith growth subject to zero-flux boundary condition are investigated analytically and numerically. The asymptotic stability of the positive equilibrium and the existence of Hopf bifurcation around the positive equilibrium are shown; the conditions of Turing instability are obtained. And with the help of numerical simulations, it is found that the model exhibits complex pattern replication: stripes, spots-stripes mixtures, and spots Turing patterns.
\end{abstract}

\section{Introduction}

The problem of pattern formation is, perhaps, the most challenging in modern ecology, biology, chemistry, and many other fields of science [1]. Patterns generated in abiotically homogeneous environments are particularly interesting because they require an explanation based on the individual behavior of organisms. They are commonly called "emergent patterns," because they emerge from interactions in spatial scales that are much larger than the characteristic scale of individuals [2].

Turing [3] showed how the coupling of reaction and diffusion can induce instability and pattern formation. Turing's revolutionary idea was that the passive diffusion could interact with chemical reaction in such a way that even if the reaction by itself has no symmetry-breaking capabilities, diffusion can destabilize the symmetry so that the system with diffusion can have them. Segel and Jackson [4] first used reaction-diffusion system to explain pattern formation in ecological context based upon the seminal work by Turing [3]. Since then, a lot of studies have been devoted to spatiotemporal patterns which were produced by reactiondiffusion predator-prey, models with either a prey-dependent or a ratio-dependent predator functional response, for example, $[1,2,5-20]$ and references cited therein.
Recently, there is a growing explicit biological and physiological evidence [21-23] that in many situations, especially, when the predator has to search for food (and therefore has to share or compete for food), a more suitable general predatorprey theory should be based on the so-called ratio-dependent function which can be roughly stated as that the per capital predator growth rate should be a function of the ratio of prey to predator abundance, and so would be the so-called predator functional responses [24]. This is supported by numerous fields and laboratory experiments and observations $[25,26]$. In [24], the authors investigated the effect of time delays on the stability of the model and discussed the local asymptotic stability and the Hopf bifurcation. Liang and Pan [27] have studied the local and global asymptotic stability of the coexisting equilibrium point and obtained the conditions for Poincare-Andronov-Hopf-bifurcating periodic solution. M. Banerjee and S. Banerjee [28] have studied the local asymptotic stability of the equilibrium point and obtained the conditions for the occurrence of Turing-Hopf instability for reaction-diffusion model. It is shown that prey and predator populations exhibit spatiotemporal patterns resulting from temporal oscillation of both the population and spatial instability.

Besides, in [29], Smith has shown that the logistic equation is not realistic for a food-limited population under 
the effects of environmental toxicants and established a new growth function-Smith growth function. And it has been proposed by several authors [29-34] for the dynamics of a population where the growth limitations are based on the proportion of available resources not utilized. However, pattern formation in the case of Holling-Tanner type predatorprey models with ratio-dependent functional response and Smith growth still remains an interesting area of research.

In this present work, we will focus on the ratio-dependent Holling-Tanner model with Smith growth for predator-prey interaction where random movement of both species is taken into account. The rest of the paper is organized as follows. In Section 2, we establish the ratio-dependent Holling-Tanner predator-prey model with Smith growth and study the local asymptotic stability of the positive equilibrium, existence of Hopf bifurcation around the positive equilibrium, and the conditions for the occurrence of Turing instability. In Section 3, we present and discuss the results of pattern formation via numerical simulation, which is followed by the last section, that is, conclusions and discussions.

\section{The Model and the Linear Stability Analysis}

2.1. The Model. In this paper, we rigorously consider the radio-dependent Holling-Tanner predator-prey model with Smith growth taking the form:

$$
\begin{aligned}
& \frac{d u}{d t}=\frac{r u(K-u)}{K+c u}-\frac{m u v}{u+a v}, \\
& \frac{d v}{d t}=s v\left(1-\frac{h v}{u}\right)
\end{aligned}
$$

where $u(t)$ and $v(t)$ stand for prey and predator population (density) at any instant of time t. $r, K, m, a, s, h$ are positive constants that stand for prey intrinsic growth rate, carrying capacity, capturing rate, half capturing saturation constant, predator intrinsic growth rate, conversion rate of prey into predators biomass, respectively. And $r / c$ is the replacement of mass in the population at $K$. The model with Smith growth takes into account both environmental and food chain effects of toxicant stress.

From the standpoint of biology, we are interested only in the dynamics of model (1) in the closed first quadrant $\mathbb{R}_{+}^{2}=$ $\{(u, v): u \geq 0, v \geq 0\}$. Thus, we consider only the biologically meaningful initial conditions

$$
u(0)>0, \quad v(0)>0,
$$

which are continuous functions due to their biological sense.

Straightforward computation shows that model (1) is continuous and Lipschitzian in $\mathbb{R}_{+}^{2}$ if we redefine

$$
\frac{d u}{d t}=\frac{d v}{d t}=0, \quad \text { if }(u, v)=(0,0)
$$

Hence, the solution of model (1) with positive initial conditions exists and is unique.
Also considering the spatial dispersal and environmental heterogeneity, in this paper we study the diffusive HollingTanner model obtained from the temporal model (1) by incorporating diffusion terms as follows:

$$
\begin{aligned}
& \frac{\partial u}{d t}=d_{1} \Delta u+r u \frac{K-u}{K+c u}-\frac{m u v}{u+a v} \\
& \frac{\partial v}{d t}=d_{2} \Delta v+s v\left(1-\frac{h v}{u}\right)
\end{aligned}
$$

where the nonnegative constants $d_{1}$ and $d_{2}$ are the diffusion coefficients of $u$ and $v$, respectively. $\Delta=\left(\partial^{2} / \partial x^{2}\right)+\left(\partial^{2} / \partial y^{2}\right)$, the usual Laplacian operator in two-dimensional space, is used to describe the Brownian random motion.

Model (4) is to be analyzed under the following nonzero initial conditions:

$$
u(x, y, 0)>0, \quad v(x, y, 0)>0, \quad(x, y) \in \Omega,
$$

and zero-flux boundary conditions:

$$
\frac{\partial u}{\partial v}=\frac{\partial v}{\partial v}=0, \quad(x, y) \in \partial \Omega, t>0,
$$

where $\Omega \subset \mathbb{R}^{2}$ is a bounded domain with a smooth boundary $\partial \Omega$ and $\nu$ is the outward unit normal vector on $\partial \Omega$. The zero-flux boundary condition indicates that predator-prey system is self-contained with zero population flux across the boundary.

2.2. The Stability of the Nonspatial Model (1). In this subsection, we restrict ourselves to the stability analysis of the nonspatial model (1). It is easy to verify that model (1) has a trivial equilibrium point $E_{0}=(K, 0)$. Simple computation shows that if $m<r(a+h)$, model (1) possess a unique positive equilibrium, denoted by $E^{*}=\left(u^{*}, v^{*}\right)$, where

$$
u^{*}=\frac{K(a r+h r-m)}{a r+c m+h r}, \quad v^{*}=\frac{1}{h} u^{*} .
$$

The Jacobian matrix at $E_{0}=(K, 0)$ is

$$
J_{0}=\left(\begin{array}{cc}
-\frac{r}{1+c} & -m \\
0 & s
\end{array}\right) \text {. }
$$

Clearly, $E_{0}=(K, 0)$ is a saddle point.

In the following, we will discuss the stability of the positive equilibrium $E^{*}$ of model (1). The Jacobian matrix at $E^{*}$ is given by

$$
J^{*}=\left(\begin{array}{cc}
a_{1} & a_{2} \\
\frac{s}{h} & -s
\end{array}\right),
$$

where

$$
\begin{gathered}
a_{1}=\frac{m(a r+c m+2 h r-a c r)-r^{2}(a+h)^{2}}{r(1+c)(a+h)^{2}}, \\
a_{2}=-\frac{m h^{2}}{(a+h)^{2}} .
\end{gathered}
$$


Then we can get

$$
\begin{aligned}
& \operatorname{det}\left(J^{*}\right) \\
& =s\left(\frac{a_{2}}{h}-a_{1}\right) \\
& =\frac{s(a r+h r-m)(a r+h r+c m)}{r(1+c)(a+h)^{2}}>0, \\
& \operatorname{tr}\left(J^{*}\right) \\
& =a_{1}-s \\
& =\frac{m(a r+c m+2 h r-a c r)-r(a+h)^{2}(r+s(1+c))}{r(1+c)(a+h)^{2}} .
\end{aligned}
$$

Theorem 1. (i) The positive equilibrium $E^{*}=\left(u^{*}, v^{*}\right)$ is locally asymptotically stable if and only if

$$
m(a r+c m+2 h r-a c r)<r(a+h)^{2}(r+s(1+c)) .
$$

(ii) The positive equilibrium $E^{*}=\left(u^{*}, v^{*}\right)$ is unstable if and only if

$$
m(a r+c m+2 h r-a c r)>r(a+h)^{2}(r+s(1+c)) .
$$

(iii) The model enters into a Hopf-bifurcation around $E^{*}=$ $\left(u^{*}, v^{*}\right)$ at $s=s^{*}$, where $s^{*}$ satisfies the equality

$$
s^{*}=\frac{m(a r+c m+2 h r-a c r)-r^{2}(a+h)^{2}}{r(1+c)(a+h)^{2}} .
$$

Proof. (i) If $m(a r+c m+2 h r-a c r)<r(a+h)^{2}(r+s(1+$ $c)$ ), then $\operatorname{tr}\left(J^{*}\right)<0$. Thus, the equilibrium point $E^{*}$ is locally asymptotically stable, similar to the proof of (ii).

(iii) A Hopf bifurcation occurs if and only if there exists a $s=s^{*}$ such that

$$
\operatorname{tr}\left(J^{*}\right)=a_{1}-s^{*}=0, \quad \frac{d}{d s} \operatorname{Re}(\lambda(s))_{s=s^{*}} \neq 0,
$$

where $\lambda$ is a root of the characteristic equation of $J^{*}$ :

$$
\lambda^{2}-\operatorname{tr}\left(J^{*}\right) \lambda+\operatorname{det}\left(J^{*}\right)=0
$$

The condition $s^{*}=a_{1}$ gives $\operatorname{tr}\left(J^{*}\right)=0$. Thus for $s=s^{*}$, both eigenvalues will be purely imaginary and there are no other eigenvalues with negative real part. Now we verify the transversality condition $\left.(d / d s) \operatorname{Re}(\lambda(s))\right|_{s=s^{*}} \neq 0$.

Substituting $\lambda=\alpha+i \beta$ into the equation $\lambda^{2}-\operatorname{tr}\left(J^{*}\right) \lambda+$ $\operatorname{det}\left(J^{*}\right)=0$ and separating real and imaginary parts we obtain

$$
\begin{gathered}
\alpha^{2}-\beta^{2}-\alpha \operatorname{tr}\left(J^{*}\right)+\operatorname{det}\left(J^{*}\right)=0, \\
2 \alpha \beta-\beta \operatorname{tr}\left(J^{*}\right)=0 .
\end{gathered}
$$

Differentiating (17) both sides with respect to $s$, we get

$$
\begin{aligned}
& \varphi \frac{d \alpha}{d s}-2 \beta \frac{d \beta}{d s}=\gamma, \\
& 2 \beta \frac{d \alpha}{d s}+\varphi \frac{d \beta}{d s}=\phi,
\end{aligned}
$$

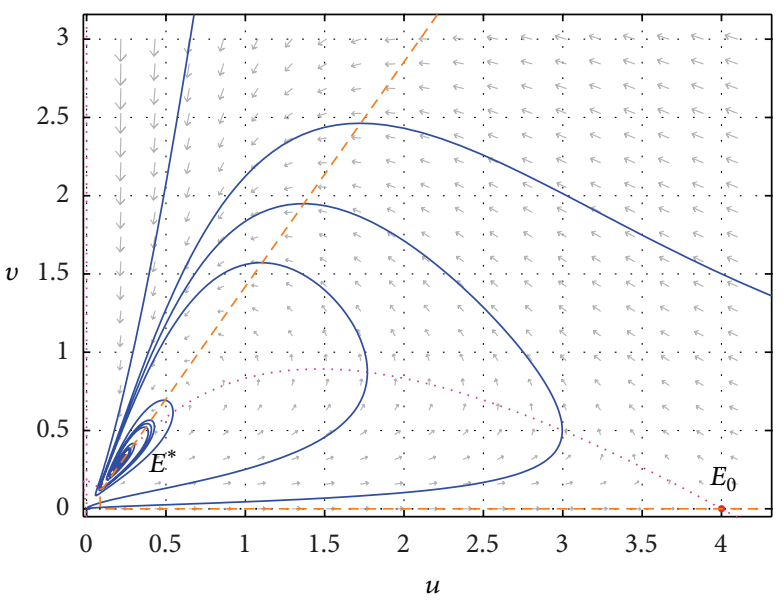

FIGURE 1: Phase portraits of model (1). The parameters are taken as $r=1, m=1, a=0.4, K=4, h=0.7, c=0.8, s=0.55 . E_{0}=(K, 0)$ is a saddle point. $E^{*}=(0.21053,0.30075)$ is locally asymptotically stable. The dashed curve is the $u$-nullcline, and the dotted vertical line is the $v$-nullcline.

where $\varphi=2 \alpha-\operatorname{tr}\left(J^{*}\right), \phi=\alpha\left(d\left(\operatorname{tr}\left(J^{*}\right)\right) / d s\right)-(d(\operatorname{det}$ $\left.\left.\left(J^{*}\right)\right) / d s\right), \gamma=\beta\left(d\left(\operatorname{det}\left(J^{*}\right)\right) / d s\right)$. Thus, we obtain

$$
\left.\frac{d}{d s} \operatorname{Re}(\lambda(s))\right|_{s=s^{*}}=\left.\frac{\gamma \varphi+2 \beta \phi}{\varphi^{2}+4 \beta^{2}}\right|_{s=s^{*}} \neq 0,
$$

which verify the transversality condition. Hence, the system undergoes a Hopf bifurcation at $E^{*}$ as $s$ passes through the value $s^{*}$. This ends the proof.

In Figure 1, we show the phase portraits of (1) with $r=1$, $m=1, a=0.4, K=4, h=0.7, c=0.8$, and $s=0.55$. The horizontal axis is the prey population $u$, and the vertical axis is the predator population $v$. The dashed curve is the $u$ nullcline, and the dotted vertical line is the $v$-nullcline. It is easy to see that the equilibrium $E_{0}=(4,0)$ is a saddle and $E^{*}=(0.21053,0.30075)$ is locally asymptotically stable.

Figure 2 illustrates a Hopf-bifurcation situation of the model around $E^{*}=(0.21053,0.30075)$ for $s=s^{*}=$ 0.4912764003. In this case, limit cycle arising through Hopf bifurcation is a stable limit cycle which attracts all trajectories starting from a point in the interior of first quadrant.

2.3. The Stability of the Spatial Model (4). In this subsection, we will focus on the effect of diffusion on the model system about the positive equilibrium.

Now, we study the nonlinear evolution of a perturbation

$$
U(x, t)=u(x, t)-u^{*}, \quad V(x, t)=v(x, t)-v^{*}
$$

around $E^{*}=\left(u^{*}, v^{*}\right)$. The corresponding linearized model (4) then takes the form

$$
\begin{gathered}
\frac{\partial U}{d t}=d_{1} \Delta U+a_{1} U+a_{2} V, \\
\frac{\partial V}{d t}=d_{2} \Delta V+\frac{s}{h} U-s V,
\end{gathered}
$$

where $a_{1}, a_{2}$ are defined the same as (10). 


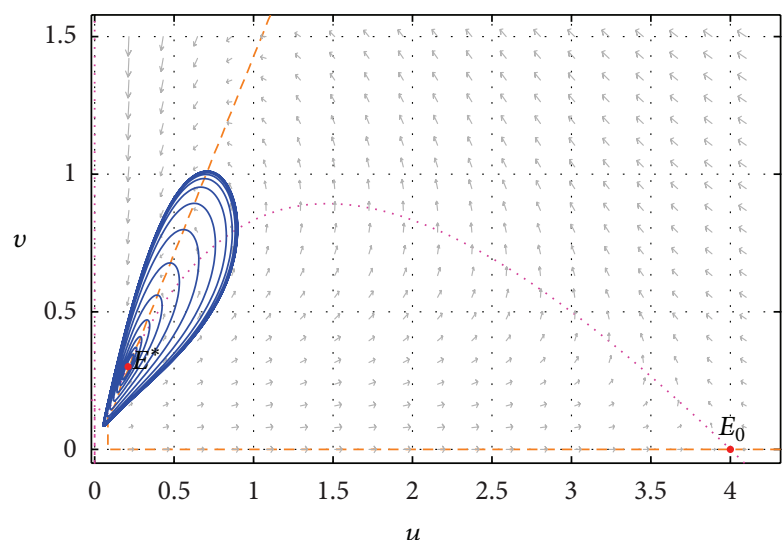

FIgURE 2: Phase portraits of model (1). The parameters are taken as $r=1, m=1, a=0.4, K=4, h=0.7, c=0.8, s=$ 0.4912764003 . Model (1) enters into Hopf bifurcation around $E^{*}=$ $(0.21053,0.30075)$, and there is a limit cycle. The dashed curve is the $u$-nullcline, and the dotted vertical line is the $v$-nullcline.

We use $[\cdot, \cdot]$ to denote a column vector, and let

$$
\mathbf{w}(x, t)=[U(x, t), V(x, t)] .
$$

Let $\mathbf{q}=\left(q_{1}, q_{2}\right) \in \Omega$ and

$$
e_{\mathbf{q}}(x)=\prod_{i=1}^{2} \cos \left(q_{i} x_{i}\right)
$$

Then $\left\{e_{\mathbf{q}}(x)\right\}_{\mathbf{q} \in \Omega}$ forms a basis of the space of functions in $\mathbb{R}^{2}$ that satisfy zero-flux boundary conditions. We look for a normal mode corresponding to model (21) as following form:

$$
\mathbf{w}(x, t)=\mathbf{r}_{\mathbf{q}} \exp \left(\lambda_{\mathbf{q}} t\right) e_{\mathbf{q}}(x),
$$

where $\mathbf{r}_{\mathbf{q}}$ is a vector depending on q. Plugging (24) into model (21) yields

$$
\lambda_{\mathbf{q}} \mathbf{r}_{\mathbf{q}}=\left(\begin{array}{cc}
-d_{1} q^{2}+a_{1} & a_{2} \\
\frac{s}{h} & -d_{2} q^{2}-s
\end{array}\right) \mathbf{r}_{\mathbf{q}},
$$

where $q^{2}=q_{1}^{2}+q_{2}^{2}$. A nontrivial normal mode can be obtained by setting

$$
\operatorname{det}\left(\begin{array}{cc}
\lambda_{\mathbf{q}}+d_{1} q^{2}-a_{1} & -a_{2} \\
-\frac{s}{h} & \lambda_{\mathbf{q}}+d_{2} q^{2}+s
\end{array}\right)=0 .
$$

This leads to the following dispersion formula for $\lambda_{\mathbf{q}}$ :

$$
\lambda_{\mathbf{q}}^{2}+\rho_{1} \lambda_{\mathbf{q}}+\rho_{2}=0
$$

where

$$
\begin{gathered}
\rho_{1}=\left(d_{1}+d_{2}\right) q^{2}-a_{1}+s, \\
\rho_{2}=d_{1} d_{2} q^{4}+\left(d_{1} s-d_{2} a_{1}\right) q^{2}+s\left(\frac{a_{2}}{h}-a_{1}\right) .
\end{gathered}
$$

Mathematically speaking, a positive equilibrium $E^{*}$ of model (4) is Turing unstable, which means that it is an asymptotically stable steady-state solution of the model (1) without diffusion but is unstable with respect to the solutions of the model (4) with diffusion.

Therefore, the Turing instability sets in when at least one of the following conditions is violated:

$$
\rho_{1}<0, \quad \rho_{2}>0 .
$$

But it is evident that $\rho_{1}<0$ is not violated if $s-a_{1}<0$. Hence only the violation of condition $\rho_{2}>0$ gives rise to diffusion instability. As a consequence, a necessary condition is

$$
d_{1} s<d_{2} a_{1} .
$$

Otherwise $\rho_{2}>0$ for all $q>0$. For instability we must have $\rho_{2}<0$ for some $q>0$, and we notice that $\rho_{2}$ achieves its minimum:

$$
\min _{p \in \mathbb{R}^{+}} \rho_{2}=-\frac{\left(d_{1} s-d_{2} a_{1}\right)^{2}}{4 d_{1} d_{2}}+s\left(\frac{a_{2}}{h}-a_{1}\right),
$$

at the critical value $p_{c}^{2}>0$ when

$$
p_{c}^{2}=\frac{d_{2} a_{1}-d_{1} s}{2 d_{1} d_{2}}>0 .
$$

Summarizing the previous calculation, we conclude the following theorem.

Theorem 2. Assume that

$$
\text { (A1) } \begin{aligned}
& m(a r+c m+2 h r-a c r)<r(a+h)^{2}(r+s+c s), \\
\text { (A2) } & r(a+h)^{2}\left(d_{1} s(1+c)+d_{2} r\right) \\
& +2 \sqrt{d_{1} d_{2} r s(1+c)(a+h)^{2}(a r+h r-m)(a r+h r+c m)} \\
< & d_{2} m(a r+c m+2 h r-a c r) .
\end{aligned}
$$

Then the positive equilibrium $E^{*}$ of model (4) is Turing unstable.

From Theorem 2, we can know that there is Turing instability in model (4) if conditions (A1) and (A2) hold. In this situation, the solutions to model (4) may be unstable and Turing patterns can emerge in the model.

\section{Turing Pattern Formation}

In this section, we perform extensive numerical simulations of the spatially extended model (4) in two-dimensional space, and the qualitative results are shown here. All our numerical simulations employ the zero-flux boundary conditions with a system size of $100 \times 100$. Other parameters are set as

$$
\begin{aligned}
& r=1, \quad m=1, \quad a=0.4, \quad K=4, \\
& h=0.7, \quad c=0.8, \quad d_{1}=0.025, \quad d_{2}=1 .
\end{aligned}
$$

The numerical integration of model (4) is performed by using a finite difference approximation for the spatial derivatives and an explicit Euler method for the time integration 


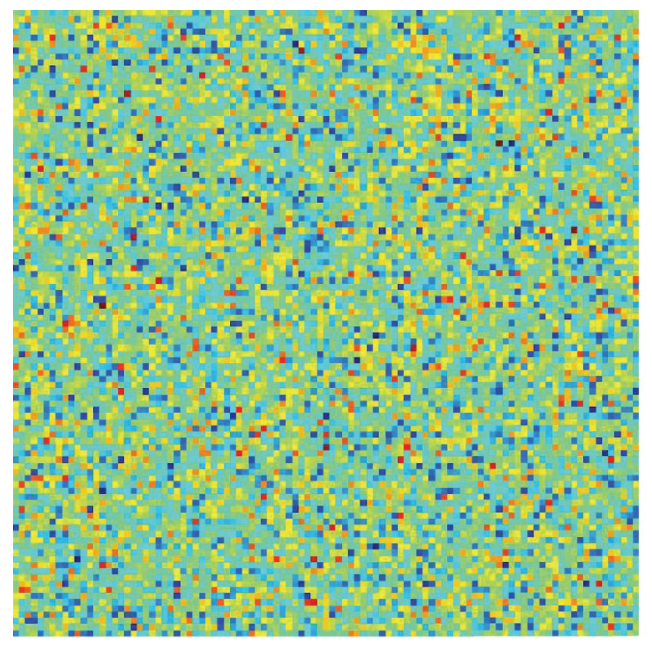

(a)

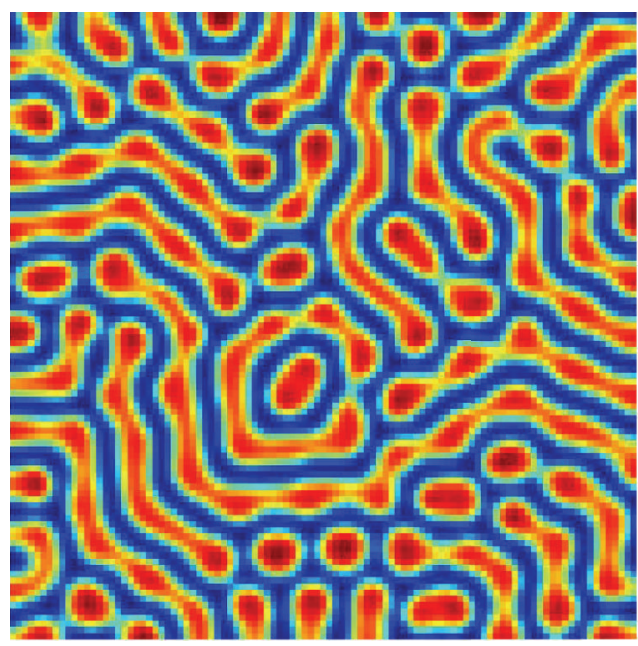

(c)
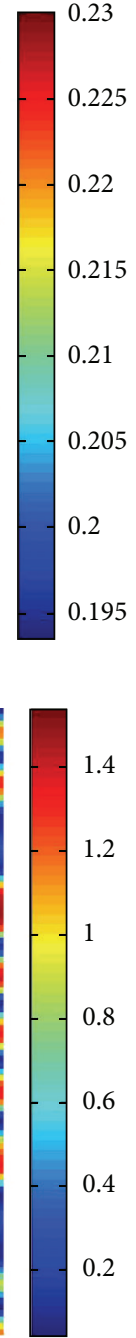

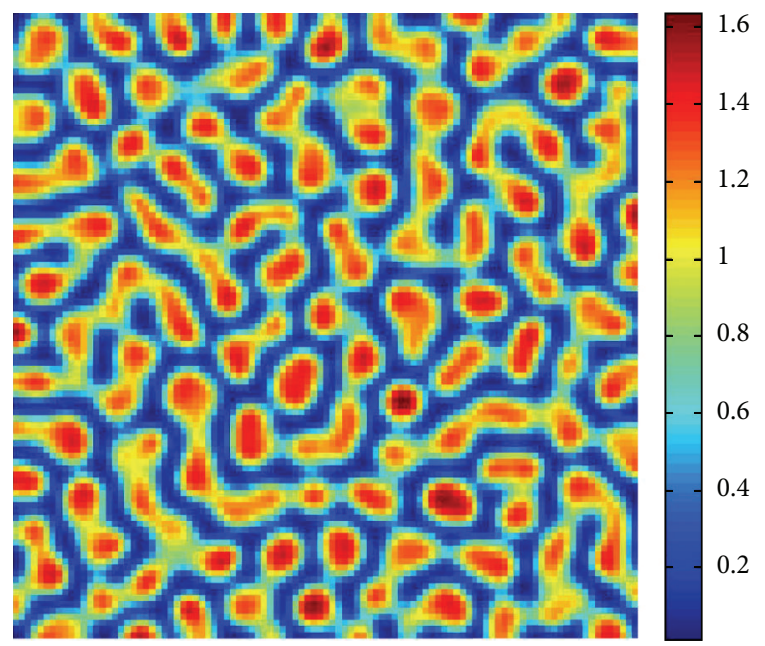

(b)

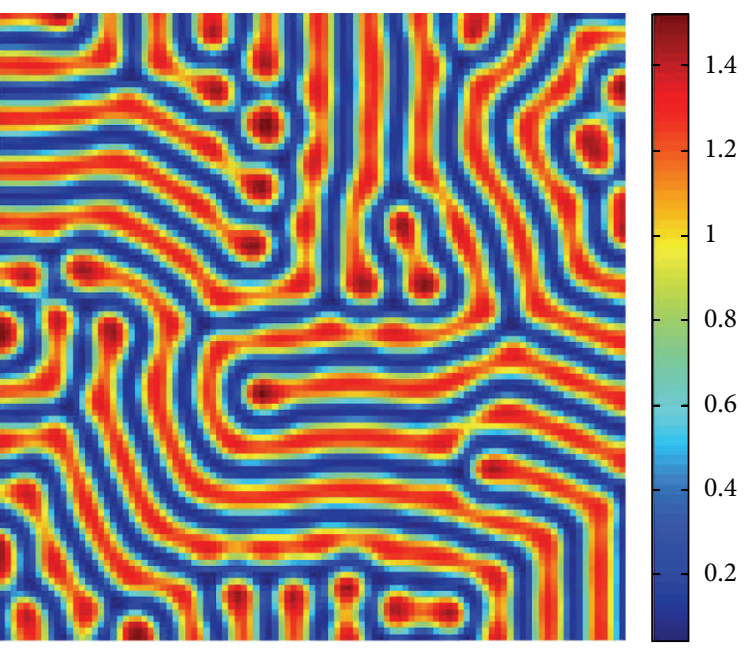

(d)

FIGURE 3: Stripes pattern formation for model (4) by taking $s=1$. Other parameters are fixed as (33). Times: (a) 0; (b) 50; (c) 250; (d) 2500.

[35] with a time step size of $1 / 100$. The initial condition is always a small amplitude random perturbation around the positive constant steady-state solution $E^{*}=\left(u^{*}, v^{*}\right)$. After the initial period during which the perturbation spread, the model either goes to a time-dependent state or to an essentially steady-state solution (time independent).

We use the standard five-point approximation [36] for the 2D Laplacian with the zero-flux boundary conditions. More precisely, the concentrations $\left(u_{i, j}^{n+1}, v_{i, j}^{n+1}\right)$ at the moment $(n+$ $1) \tau$ at the mesh position $\left(x_{i}, y_{j}\right)$ are given by

$$
\begin{aligned}
& u_{i, j}^{n+1}=u_{i, j}^{n}+\tau d_{1} \Delta_{e} u_{i, j}^{n}+\tau f\left(u_{i, j}^{n}, v_{i, j}^{n}\right), \\
& v_{i, j}^{n+1}=v_{i, j}^{n}+\tau d_{2} \Delta_{e} v_{i, j}^{n}+\tau g\left(u_{i, j}^{n}, v_{i, j}^{n}\right),
\end{aligned}
$$

with the Laplacian defined by

$$
\Delta_{e} u_{i, j}^{n}=\frac{u_{i+1, j}^{n}+u_{i-1, j}^{n}+u_{i, j+1}^{n}+u_{i, j-1}^{n}-4 u_{i, j}^{n}}{e^{2}}
$$

where $f(u, v)=(r u(K-u) /(K+c u))-(m u v /(u+a v))$, $g(u, v)=s v(1-(h v / u))$, and the space step size $e=1 / 3$.

In the numerical simulations, different types of dynamics are observed and it is found that the distributions of predator and prey are always of the same type. Consequently, we can restrict our analysis of pattern formation to one distribution. In this section, we show the distribution of prey $u$, for instance. We have taken some snapshots with red (blue) corresponding to the high (low) value of prey $u$.

Now, we show the Turing patterns for the different values of the control parameter $s$. Via numerical simulations, one can see that the model dynamics exhibits spatiotemporal complexity of pattern formation, including stripes, stripesspots mixtures, and spots Turing patterns.

In Figure 3, with $s=1$, starting with a homogeneous state $E^{*}=(0.21,0.3)$ (cf. Figure $\left.3(\mathrm{a})\right)$, the random perturbations lead to the formation to stripes spots (cf. Figure 3(c)), and the latter random perturbations make these spots decay, ending with the time-independent stripes pattern (cf. Figure 3(d)). 


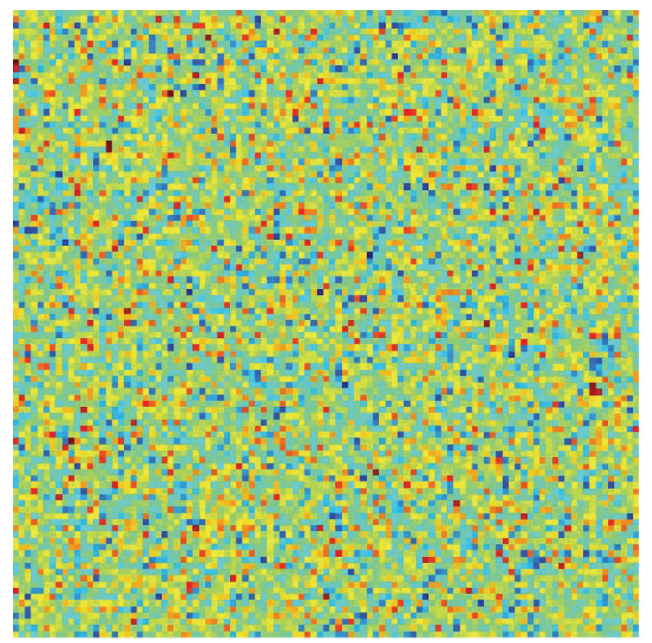

(a)

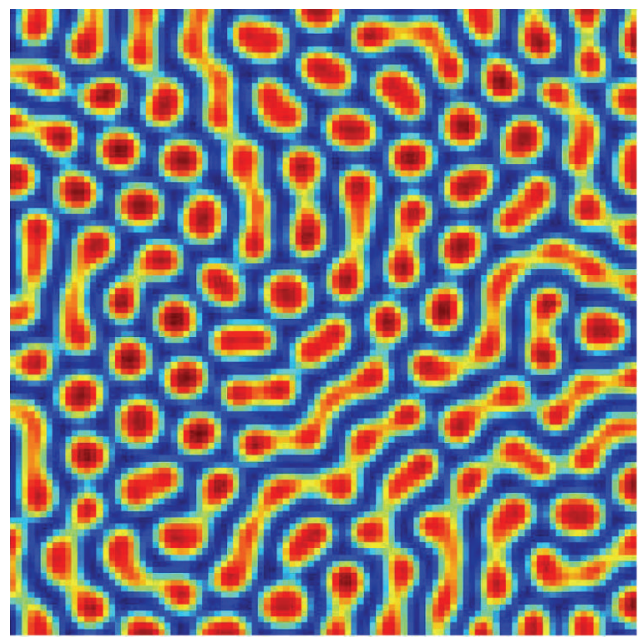

(c)
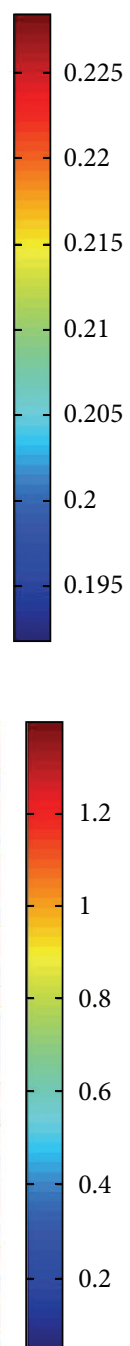

0.2

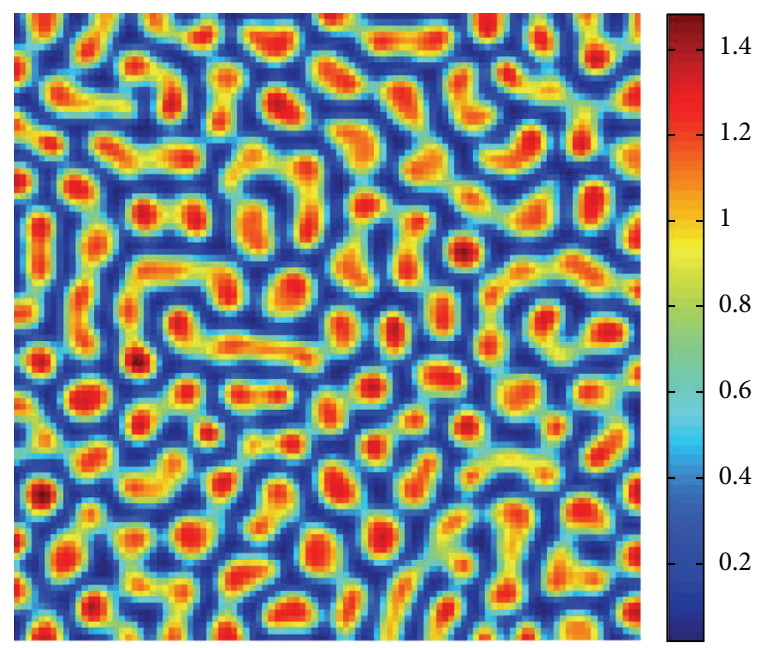

(b)

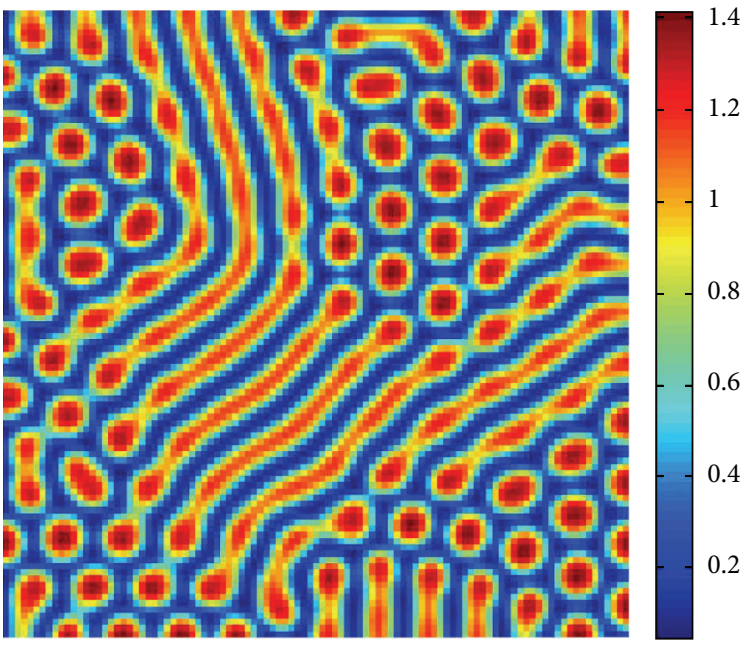

(d)

FIgURE 4: Stripes-spots mixtures pattern formation for model (4) by taking $s=1.375$. Other parameters are fixed as (33). Times: (a) 0 ; (b) 50; (c) 250; (d) 2500 .

In Figure 4, with $s=1.375$, we show the stripes-spots mixtures pattern for model (4).

Figure 5 shows the time process of spots pattern formation of prey $u$ for $s=2.5$. In this case, the pattern takes a long time to settle down, starting with a homogeneous state $E^{*}=$ $(0.21,0.3)$ (cf. Figure 5(a)), and the random perturbations lead to the formation of stripes and spots (cf. Figure 5(b)), ending with spots only (cf. Figure $5(\mathrm{~d})$ ) - the prey $u$ is isolated zones with high population density, and the remainder region is of low density.

From Figure 3 to Figure 5, we can see that, on increasing the control parameter $s$ from 1 to 2.5 , the pattern sequence "stripe $\rightarrow$ stripes-spots mixtures $\rightarrow$ spots" can be observed.

\section{Conclusions and Remarks}

In summary, in this paper, we have investigated the spatiotemporal dynamics of a diffusive predator-prey model where the interaction between prey and predator follows Holling-Tanner formulation with ratio-dependent functional response and Smith growth. The value of this study is threefold. First, it presents the conditions for the stability of the equilibrium and the existence of Hopf bifurcation for the nonspatial model. Second, it rigorously proves Turing instability by linear stability analysis for the spatial model. Third, it illustrates the Turing pattern formation via numerical simulations, which shows that the spatial model dynamics exhibits complex pattern replication.

By a series of numerical simulations, we find that the spatial model (4) has rich Turing pattern replications, such as stripes, stripes-spots mixtures, and spots patterns. In the viewpoint of population ecology, in the case of stripe pattern (cf. Figure 3), the prey $u$ is the isolated "stripes-like region" with high density, and the remainder stripes-like region is of low density. And in the case of spots pattern (cf. Figure 5), the prey $u$ is the isolated "cycle region" with high density, and the 


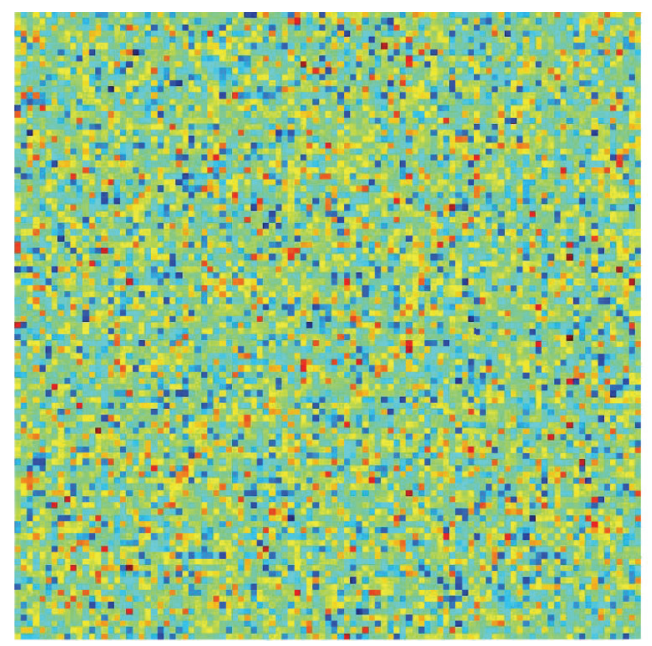

(a)

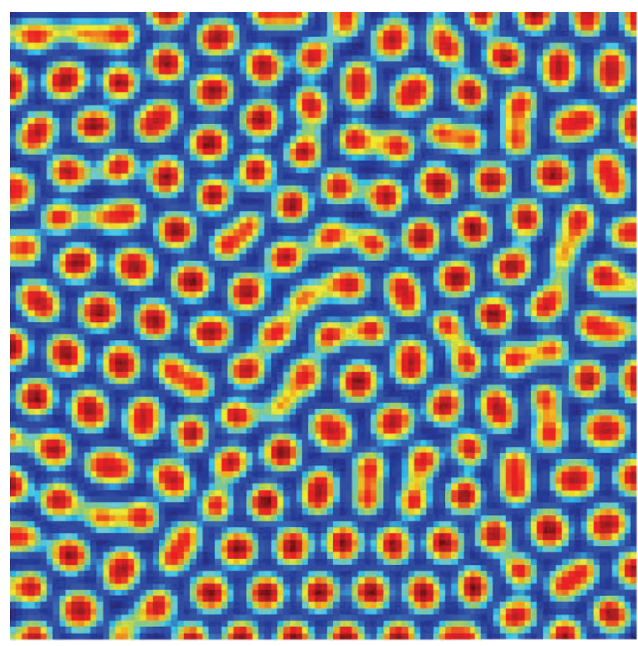

(c)
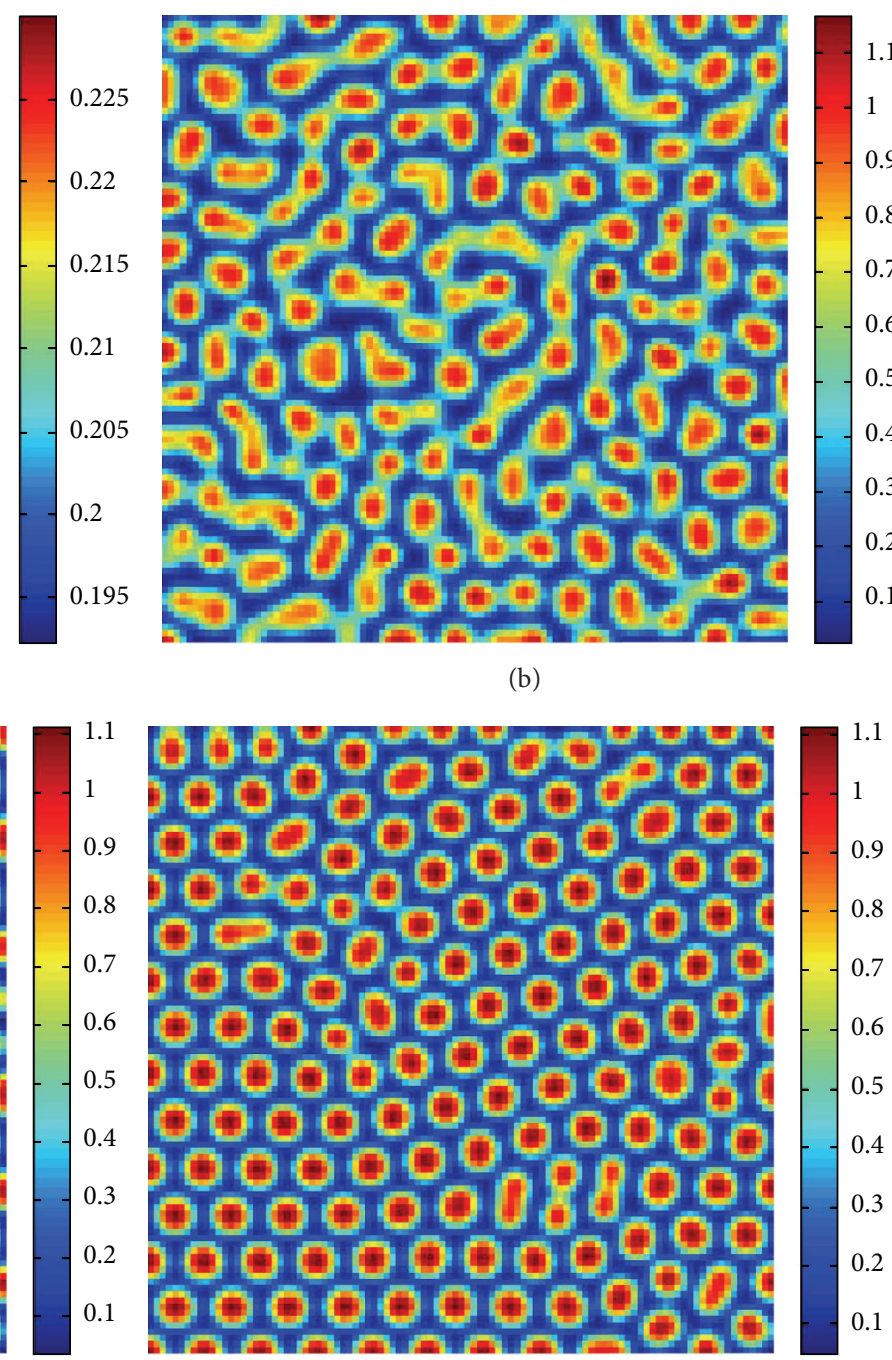

(d)

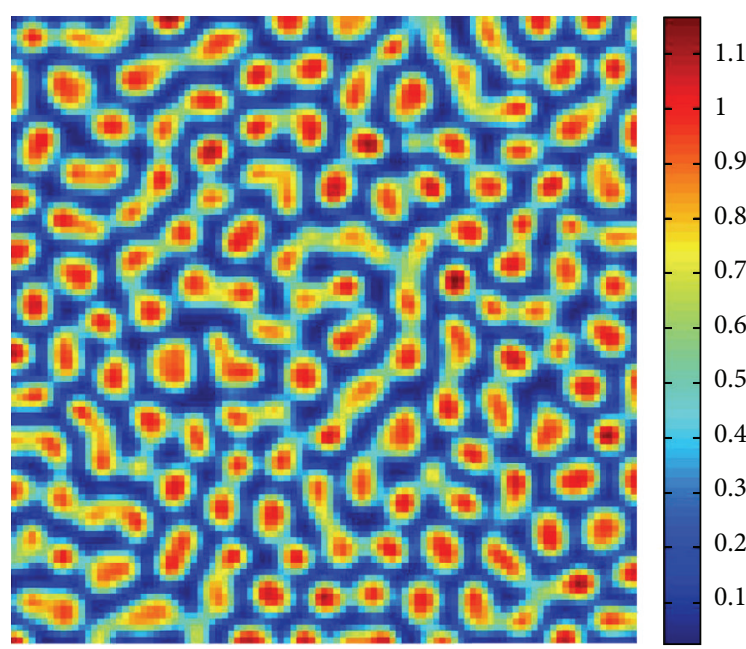

(b)

FIGURE 5: Spots pattern formation for model (4) by taking $s=2.5$. Other parameters are fixed as (33). Times: (a) 0; (b) 50; (c) 250; (d) 2500.

remainder region is of low density, which is larger than the "spots" region.

\section{References}

[1] S. V. Petrovskii and H. Malchow, "A minimal model of pattern formation in a prey-predator system," Mathematical and Computer Modelling, vol. 29, no. 8, pp. 49-63, 1999.

[2] D. Alonso, F. Bartumeus, and J. Catalan, "Mutual interference between predators can give rise to turing spatial patterns," Ecology, vol. 83, no. 1, pp. 28-34, 2002.

[3] A. M. Turing, "The chemical basis of morphogenesis," Philosactions Transactions of the Royal Society of London. Series B, vol. 237, no. 641, pp. 37-72, 1952.

[4] L. A. Segel and J. L. Jackson, "Dissipative structure: an explanation and an ecological example," Journal of Theoretical Biology, vol. 37, no. 3, pp. 545-559, 1972.

[5] L. S. Luckinbill, "The effects of space and enrichment on a predator-prey system," Ecology, vol. 55, no. 5, pp. 1142-1147, 1974.

[6] S. A. Levin, "The problem of pattern and scale in ecology: the Robert H. MacArthur award lecture," Ecology, vol. 73, no. 6, pp. 1943-1967, 1992.

[7] M. Pascual, "Diffusion-induced chaos in a spatial predator-prey system," Proceedings of the Royal Society B, vol. 251, no. 1330, pp. $1-7,1993$.

[8] A. B. Medvinsky, S. V. Petrovskii, I. A. Tikhonova, H. Malchow, and B.-L. Li, "Spatiotemporal complexity of plankton and fish dynamics," SIAM Review, vol. 44, no. 3, pp. 311-370, 2002.

[9] J. D. Murray, Mathematical Biology. II: Spatial Models and Biomedical Applications, vol. 18 of Interdisciplinary Applied Mathematics, Springer, New York, NY, USA, 3rd edition, 2003.

[10] A. B. Peet, P. A. Deutsch, and E. Peacock-López, "Complex dynamics in a three-level trophic system with intraspecies interaction," Journal of Theoretical Biology, vol. 232, no. 4, pp. 491-503, 2005.

[11] D. A. Griffith and P. R. Peres-Neto, "Spatial modeling in ecology: the flexibility of eigenfunction spatial analyses," Ecology, vol. 87, no. 10, pp. 2603-2613, 2006. 
[12] I. G. Pearce, M. A. J. Chaplain, P. G. Schofield, A. R. A. Anderson, and S. F. Hubbard, "Modelling the spatio-temporal dynamics of multi-species host-parasitoid interactions: heterogeneous patterns and ecological implications," Journal of Theoretical Biology, vol. 241, no. 4, pp. 876-886, 2006.

[13] W. Wang, Q.-X. Liu, and Z. Jin, "Spatiotemporal complexity of a ratio-dependent predator-prey system," Physical Review E, vol. 75, no. 5, Article ID 051913, 9 pages, 2007.

[14] W. Wang, L. Zhang, H. Wang, and Z. Li, "Pattern formation of a predator-prey system with Ivlev-type functional response," Ecological Modelling, vol. 221, no. 2, pp. 131-140, 2010.

[15] W. Wang, Y. Lin, F. Rao, L. Zhang, and Y. Tan, "Pattern selection in a ratio-dependent predator-prey model," Journal of Statistical Mechanics: Theory and Experiment, vol. 2010, Article ID P11036, 2010.

[16] W. Wang, Y. Lin, L. Zhang, F. Rao, and Y. Tan, "Complex patterns in a predator-prey model with self and cross-diffusion," Communications in Nonlinear Science and Numerical Simulation, vol. 16, no. 4, pp. 2006-2015, 2011.

[17] X. Guan, W. Wang, and Y. Cai, "Spatiotemporal dynamics of a Leslie-Gower predator-prey model incorporating a prey refuge," Nonlinear Analysis: Real World Applications, vol. 12, no. 4, pp. 2385-2395, 2011.

[18] Y. Zhu, Y. Cai, S. Yan, and W. Wang, "Dynamical analysis of a delayed reaction-diffusion predator-prey system," Abstract and Applied Analysis, vol. 2012, Article ID 323186, 23 pages, 2012.

[19] Y. Cai, W. Wang, and J. Wang, "Dynamics of a diffusive predator-prey model with additive Allee effect," International Journal of Biomathematics, vol. 5, no. 2, Article ID 1250023, 11 pages, 2012.

[20] W. Wang, Z. Guo, R. K. Upadhyay, and Y. Lin, "Pattern formation in a cross-diffusive Holling-Tanner model," Discrete Dynamics in Nature and Society, vol. 2012, Article ID 828219, 12 pages, 2012.

[21] R. Arditi, L. R. Ginzburg, and H. R. Akcakaya, "Variation in plankton densities among lakes: a case for ratio-dependent models," The American Naturalist, vol. 138, no. 5, pp. 287-1296, 1991.

[22] R. Arditi and H. Saiah, "Empirical evidence of the role of heterogeneity in ratio-dependent consumption," Ecology, vol. 73, no. 5, pp. 1544-1551, 1992.

[23] A. P. Gutierrez, "Physiological basis of ratio-dependent predator-prey theory: the metabolic pool model as a paradigm," Ecology, vol. 73, no. 5, pp. 1552-1563, 1992.

[24] T. Saha and C. Chakrabarti, "Dynamical analysis of a delayed ratio-dependent Holling-Tanner predator-prey model," Journal of Mathematical Analysis and Applications, vol. 358, no. 2, pp. 389-402, 2009.

[25] R. Arditi and L. R. Ginzburg, "Coupling in predator-prey dynamics: ratio-dependence," Journal of Theoretical Biology, vol. 139, no. 3, pp. 311-326, 1989.

[26] R. Arditi, N. Perrin, and H. Saiah, "Functional responses and heterogeneities: an experimental test with cladocerans," Oikos, vol. 60, no. 1, pp. 69-75, 1991.

[27] Z. Liang and H. Pan, "Qualitative analysis of a ratio-dependent Holling-Tanner model," Journal of Mathematical Analysis and Applications, vol. 334, no. 2, pp. 954-964, 2007.

[28] M. Banerjee and S. Banerjee, "Turing instabilities and spatiotemporal chaos in ratio-dependent Holling-Tanner model," Mathematical Biosciences, vol. 236, no. 1, pp. 64-76, 2012.
[29] F. E. Smith, "Population dynamics in Daphnia Magna and a new model for population growth," Ecology, vol. 44, pp. 651663, 1963.

[30] E. C. Pielou, An Introduction to Mathematical Ecology, John Wiley \& Sons, New York, NY, USA, 1969.

[31] T. G. Hallam and J. T. Deluna, "Effects of toxicants on populations: a qualitative approach III," Journal of Theoretical Biology, vol. 109, no. 3, pp. 411-429, 1984.

[32] K. Gopalsamy, M. R. S. Kulenović, and G. Ladas, "Environmental periodicity and time delays in a "food-limited" population model," Journal of Mathematical Analysis and Applications, vol. 147, no. 2, pp. 545-555, 1990.

[33] W. Feng and X. Lu, "On diffusive population models with toxicants and time delays," Journal of Mathematical Analysis and Applications, vol. 233, no. 1, pp. 373-386, 1999.

[34] M. Fan and K. Wang, "Periodicity in a "food-limited" population model with toxicants and time delays," Acta Mathematicae Applicatae Sinica, vol. 18, no. 2, pp. 309-314, 2002.

[35] M. R. Garvie, "Finite-difference schemes for reaction-diffusion equations modeling predator-prey interactions in MATLAB," Bulletin of Mathematical Biology, vol. 69, no. 3, pp. 931-956, 2007.

[36] A. Munteanu and R. V. Solé, "Pattern formation in noisy self-replicating spots," International Journal of Bifurcation and Chaos, vol. 16, no. 12, pp. 3679-3685, 2006. 


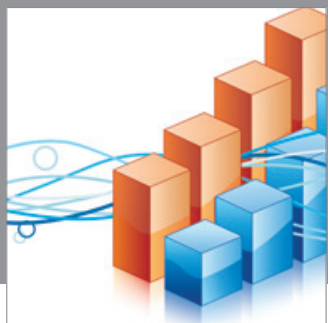

Advances in

Operations Research

mansans

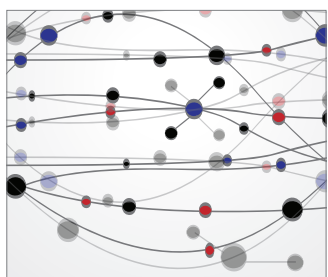

The Scientific World Journal
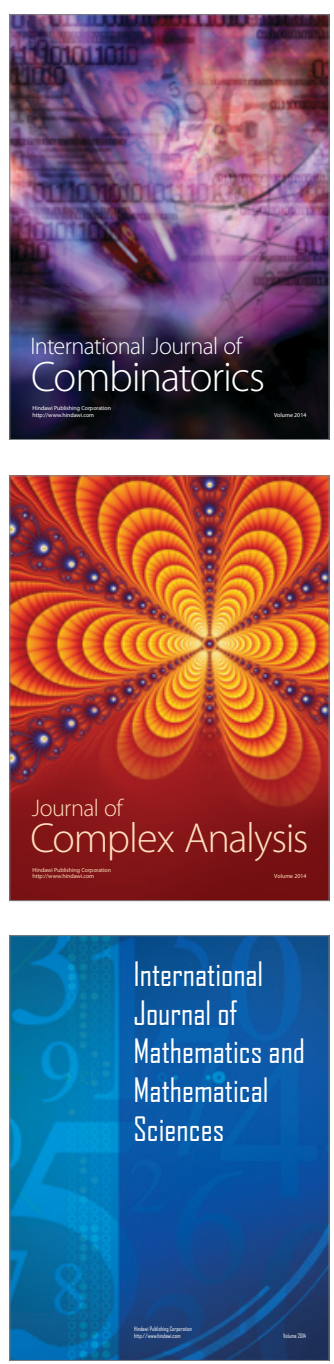
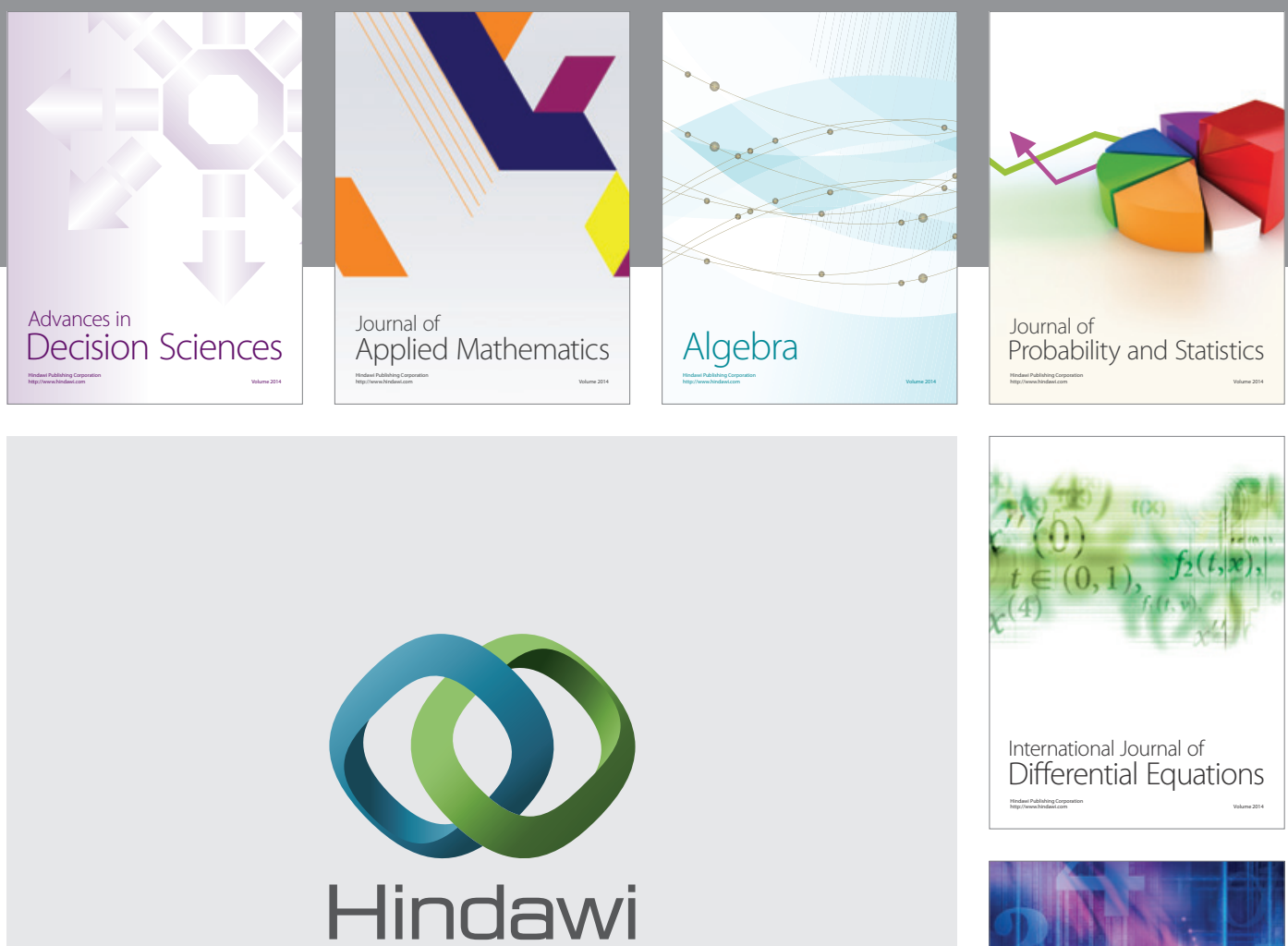

Submit your manuscripts at http://www.hindawi.com
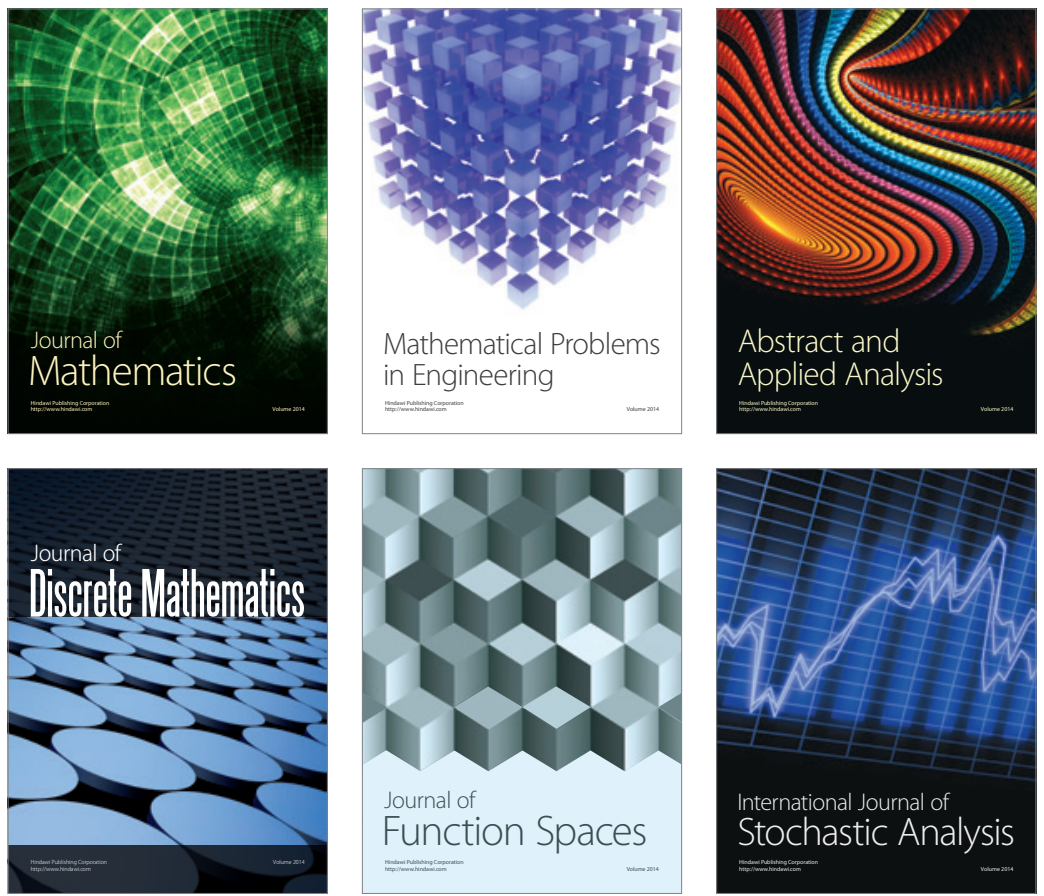

Journal of

Function Spaces

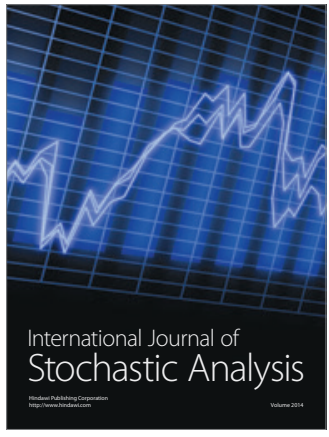

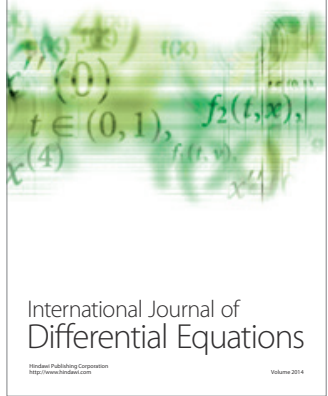
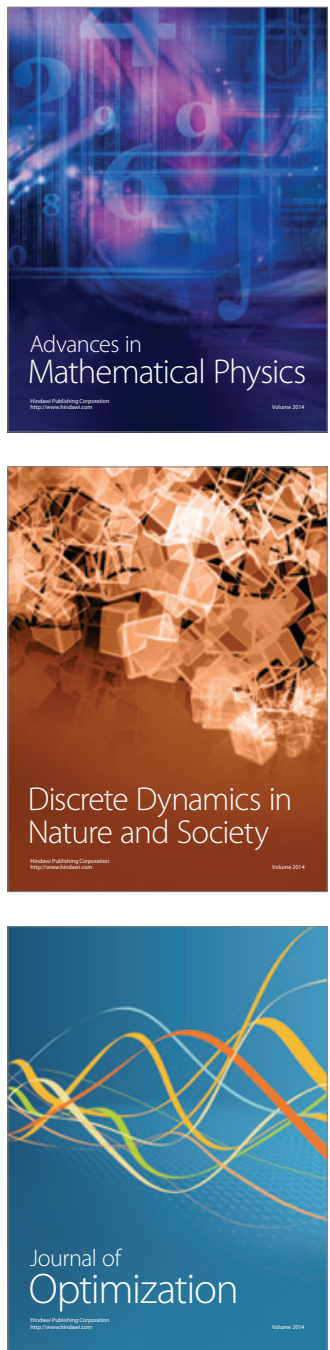